\title{
Validity of the Cloud Model of the Interstellar Medium
}

\author{
BERTRAM DONN \\ Department of Physics, Wayne State University, Detroit, Michigan
}

\section{USE OF MODEL REPRESENTATIONS}

$\mathrm{B}^{\mathrm{E}}$ ECAUSE of the extreme irregularity in distribution of interstellar matter, some idealized model must be adopted in discussing the interstellar medium. Three simple models have been considered. The original one, long-since discarded, was a uniform distribution. This has been largely replaced by the random cloud picture in which space is visualized as filled with isolated clouds, 5-10 per kpc, about $10 \mathrm{pc}$ in diameter, the array having an exponential velocity distribution with a mean of about $8 \mathrm{~km} / \mathrm{sec}^{1}$ Lastly, there is a turbulent or a random density fluctuation model. ${ }^{2-4}$

Although the random cloud hypothesis has received most attenuation from astronomers, it has been well recognized that it is an over simplified model and should be used with some caution. This point of view has been clearly stated by many workers in this field, as the following statements by Oort ${ }^{5}$ and by Minkowski, ${ }^{6}$ respectively, demonstrate.

"The whole 10 percent and 10 particles $/ \mathrm{cm}^{3}$ business is a poor approximation to reality, not much better than a guess. This distribution is very irregular. I like to warn against sticking to this picture too closely. But it has been useful and may still prove useful.

"If one looks at the central Milky Way it becomes at once obvious that the schematic picture of separate clouds of 10 parsecs diameter has little resemblance to reality. An entirely chaotic mass of dark clouds of all possible shapes and sizes appears projected on the background of stars and faint emission nebulosity. ... Although some dark clouds can be seen which may have the approximate size of the 'typical' cloud which is indicated by the Trifid nebulae, they are certainly not a prevailing feature. Instead huge complexes of clouds covering many square degrees are seen broken up into numerous irregular details . . . ."

The quotation from Oort's discussion at the last symposium is particularly significant in view of the later discussion at the present symposium on the distribution problem.

Any assumed model for the distribution of interstellar matter should not be taken as representing the true properties but rather as being a useful working

\footnotetext{
1 A. Blaauw, Bull. Astron. Soc. Neth. No. 436 (1952).

2 D. Osterbrock, Astrophys. J. 116, 164 (1952).

3 L. H. Aller, Astrophys. J. 113, 120 (1951).

4 B. Donn, Astron. J. 60, 237 (1955).

5 J. H. Oort, Gas Dynamics of Cosmic Clouds (Interscience Publishers, Inc., New York, 1955), p. 226.

${ }^{6}$ R. Minkowski, reference 5 , p. 6 .
}

hypothesis. The model is acceptable to the extent that it reproduces and explains the observations in a reasonable fashion. When the hypothetical distribution is taken over into a new problem, e.g., into the dynamics of the medium, one must consider the extent to which this use of the model is justified.

\section{VALIDITY OF THE CLOUD MODEL}

Strong support for the cloud model has been based on the similar picture for the dark matter largely developed by Ambartsumian and his associates. ${ }^{7}$ The original analysis and its elaborations have been critically examined by $\mathrm{Bok}^{8}$ who found little evidence to support any definite cloud structure for the galactic absorbing layer.

The first suggestion ${ }^{9}$ for a cloud structure in the gaseous medium was an attempt to explain the nearly linear increase of sodium $D$ line intensity with distance, although the low value of the doublet ratio indicated a high degree of saturation. This required cloud dimensions of the order of $700 \mathrm{pc}$.

Adams' observations of multiple components of interstellar lines showed there was a complex array of moving elements. It seemed highly plausible to associate these with the small clouds already postulated for the solid particles in space.

From the data on frequencies of components and their velocities, attempts were made to find the space and velocity distribution of moving elements. These are connected problems and must be treated together because of overlapping of unresolved components. Such investigations implicitly assume that frequency and velocity distributions representative of the observable region of the galaxy actually exist. If the character of the interstellar gas depends markedly on position, large scale averages do not have much meaning. This would be particularly true in regions where the matter is being stirred up by expanding HII regions and $\mathrm{O}$ associations.

The ability to fit approximately a space and velocity distribution to the observations says nothing about the validity of the result except that we have a reasonable empirical formula. This holds especially for the present case because of the limited data and problems of treating the data in a statistically sound fashion. In his

${ }^{7}$ V. A. Ambartsumian, Trans. Intern. Astron. Union 7, 452 (1950).

${ }^{8}$ B. J. Bok, Bull. Harvard Coll. Obs., No. 20, 1 (1951).

${ }^{9}$ O. C. Wilson and P. W. Merrill, Astrophys. J. 86, 44 (1937). 
paper on the velocity distribution, Blaauw ${ }^{1}$ again called attention to the limitation of the model and the care necessary in interpreting other phenomena in terms of it.

Spitzer $^{10}$ showed that the cloud picture predicts considerable scatter in the relation between intensity and doublet ratio, depending upon the degree of overlapping. His attempt to fit the observations with clouds producing saturated lines does not explain the high doublet ratios within a few hundred parsecs. In addition he found it necessary to introduce transparent clouds as well. This clearly indicates that the idealization of uniform clouds is too strong.

Probably the most difficult feature to explain is the phenomenon noted by Adams ${ }^{11}$ that the low velocity component is always the strongest. For the nearby stars there is not sufficient overlapping to explain this behavior. In this connection the extremely high frequency resolution of radio telescopes can be an important factor for obtaining additional data. ${ }^{12}$

Schlüter et $a l .^{13}$ have remarked on the difficulty of

${ }^{10}$ L. Spitzer, Jr., Astrophys. J. 108, 276 (1948).

${ }_{11}$ W. H. Adams, Astrophys. J. 109, 354 (1949).

${ }^{12}$ Hagen, Lilley, and McClain, Astrophys. J. 122, 361 (1955).

${ }^{13}$ Schlüter, Schmidt, and Stumpf, Z. Astrophys. 33, 194 (1953). explaining the intensity differences between low and high velocity components in terms of blending.

We thus find upon careful examination that the cloud concept begins to meet with difficulties even for the phenomena which it was set up to explain. This immediately suggests that great caution must be used in carrying it over to other problems of interstellar gas.

\section{ALTERNATIVE MODEL}

The writer has recently proposed that more consideration be given to a model consisting of random density fluctuation. A number of high velocity currents, about one per kiloparsec, can explain the multiple components observed by Adams. These would have much shorter lives than the separate clouds. This picture again is an idealization as the gas shows large scale deviations from randomness. However, a comparison of results for the two models would indicate which results are not sensitive to the distribution assumed and which calculations need more careful study.

The object of this paper is to call attention to the necessity of using great care in performing and interpreting theoretical calculations based on models for the distribution of interstellar matter.

\section{DISCUSSION}

G. MÜNCH, Department of Astrophysics, California Institute of Technology, Pasadena, California: The strongest argument in favor of the discrete cloud model is given by the clear separation between components of multiple interstellar lines. It is true that one could attempt to make a representation of density variations in the interstellar medium by a random function of position of some sort, and attempt to evaluate some of its characteristics. But it happens that we never can expect to have enough observational material for the characterization of this random function, especially if we consider the velocity field also to have a random nature. An enormous amount of data would be required to characterize a random function depending on six variables. The reason why I still believe that the description of the fluctuations in density and velocity in the interstellar medium is appropriately described by the discrete cloud model is-I want to emphasizepurely observational. By looking at the spectrograms, one cannot help but feel that the gas masses are separated not only in velocity but also in location. I believe that it would be extremely difficult to explain the observations in terms of some kind of currents or winds as Donn suggested. Figure 1 has some connection also with Pickelner's talk. It is a reproduction of some complex interstellar lines. I have selected cases where the separation between the components is very clear. These stars are mostly stars at high galactic latitude.
Taking for example the bottom one (HD 203664), we can see that between the strong component with small displacement and the weak component which has a displacement of about $+60 \mathrm{~km} / \mathrm{sec}$ there is no absorption within say a couple of percent. Again, in HD 215733 we see an example departing somewhat from the general rule, because it has four components which are very nearly of the same strength and clearly separated. The instrumental resolving power here begins to affect them and, if I correct formally for the instrumental resolving power, I obtain four sharp delta functions. In $\mathrm{HD}$ 93521, we have another example in which appear two lines, fairly weak, but completely split. In principle, it might be thought that by observing stars near to those showing complex lines, one would outline the extent of the masses producing a particular absorption feature. But there are no appropriate stars that close in the sky. Only in a few cases I found some relatively nearby, say less than one degree apart, which may have one component very weak and no trace of the other components. The only thing that we can say is that the distribution is very singular both in velocity and in position, but we cannot tell how sharp the variations are. I mentioned that the case of HD 215774 bears some relation to Pickelner's talk and also to van de Hulst's answer to Field's question: at high galactic latitude the dispersion in velocity of the interstellar clouds seems to be larger than it is in the galactic 


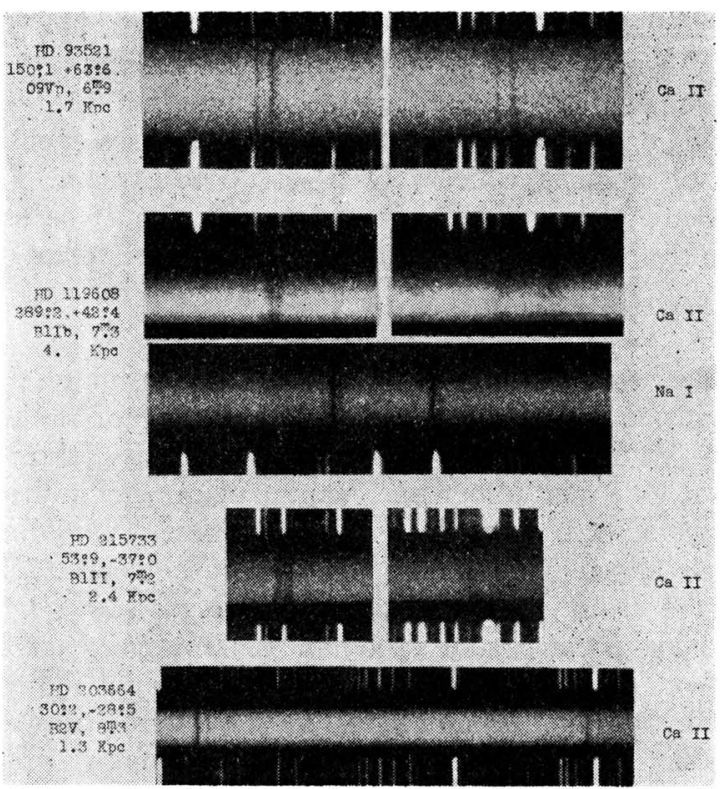

FIG. 1. Multiple interstellar lines in high galactic latitude stars. The identification HD numbers of the stars are followed by their galactic coordinates, their M.K.K. types, their apparent visual magnitudes and their distances from the sun. The top three $\mathrm{Ca}$ II spectra have original dispersion $4.5 \mathrm{~A} / \mathrm{mm}$, the $\mathrm{NaI}$ spectrum is from a $6.8 \mathrm{~A} / \mathrm{mm}$ plate and the bottom spectrum is from a $10.2 \mathrm{~A} / \mathrm{mm}$ /original. The heliocentric radial velocities in $\mathrm{km} / \mathrm{sec}$ of the various components (from left to right) are HD 93521, $-56,-35,-12$, and +4 ; HD 119608, -3 and +19 HD $215733,-57,-43,-27$, and -12 ; HD 203664, -8 and +66 .

plane-it may go up, say, to values of the order of 40 $\mathrm{km} / \mathrm{sec}$, as the $21 \mathrm{~cm}$ indicates.

BERTRAM DONN, Department of Physics, Wayne University, Detroit, Michigan: I agree it's very difficult to try, with the data available, to get a distribution in terms of a random density fluctuation. However, I think that one way in which we may be able to attempt this is with the refinements in the radio observation of the $21-\mathrm{cm}$ line where we are not restricted to stars, as we get apparatus with better angular and frequency resolution. However, I do not think that this means that we should disregard this possibility; because if we talk about collisions between clouds and calculate results from collisions between clouds which are idealized versions of the actual situation, then the results we get may be off. That is why I suggested that it might be worthwhile to try to treat the problem in the other way and see to what extent the answers agree. Also, I do not quite see why your figure indicates that we have to have clouds rather than have separate currents in a fluctuating medium.

G. MÜNCH : Another comment regarding the shape of the velocity distribution may be of some relevance. Van de Hulst emphasized the fact that, when representing the velocity distribution of interstellar clouds by some kind of smooth function, better agreement is obtained with a function which has long tails, as the Laplace distribution. There is nothing strange about the fact, mentioned by Donn earlier, that we generally observe one strong line and then a weak line separated. That just reflects the long tail of the velocity distribution. Given arbitrarily the separations and the intensities, I am certain we could build up a distribution that would explain them. However, such a velocity distribution function does not have a precise meaning in the sense of statistical mechanics, as it does not correspond to an equilibrium state. It merely represents the frequencies with which masses of various velocities are observed at a given time from a given point in space. We should not necessarily expect that it would be the same in other parts of the galaxy, especially as far as the high velocity tails are concerned.

BERTRAM DONN : The one point I made in the beginning I think applies here; viz., the fact that you can build up something from a velocity distribution does not by itself mean that this is necessarily the correct result. There are enough parameters, and we have sufficiently limited data, so that we can build up a fairly close representation. However, I do not see how, in terms of a velocity distribution, you can say that overlapping is responsible for the fact that some low velocity components are much weaker than high velocity components in other stars, because we presume on the basis of the cloud model that we have more overlapping in the strong components at low velocities. But, some lines may have a relatively high velocity and yet seem to be blends. It seems very strange that it should work out this way.

I do not say that this distribution is the complete answer. I think that this type of representation should be looked into carefully to see how far we can get with it and what the results will be.

M. P. SAVEdoff, Department of Astronomy, University of Rochester, Rochester, New York: Donn's picture seems to me to be extremely strange. I do not assume that one could walk around and tag each cloud with a number and find that they all have the same radius and density and opacity. But it seems that the clouds have a marked advantage as far as the hydrodynamics is concerned: if you put your material in clouds you get the smallest dissipation possible. I am convinced that if you have a continuous medium with velocity discontinuities, etc., the small figure of $10^{-27}$ would suddenly climb up to $10^{-23}$ or so. However, this does not mean that the cloud model is very convenient for us. The very fact that we talk in terms of clouds of small dimensions means that we have to admit the fact that these things are not gravitationally stable and are very likely to be expanding at roughly the sound velocity. Hence, the cloud model has a typical lifetime, if I remember correctly, of the order of about 10 to 20 million years, even without collisions. 